\title{
ON THE DENSITY OF PROPER EFFICIENT POINTS
}

\author{
FU WANTAO
}

(Communicated by Joseph S. B. Mitchell)

\begin{abstract}
In this paper, our aim is to discuss the density of proper efficient points. As an interesting application of the results in this paper, we want to prove a density theorem of Arrow, Barankin, and Blackwell.
\end{abstract}

In [1], Luc introduced a new concept of the proper efficient point for a set. Using some results of recession cone, Luc established efficiency conditions, especially proper efficiency and domination properties $([1,2])$. The present paper is devoted to the study of the density of proper efficient points. In detail, the set of proper efficient points for a set is dense in the set of efficient points. As an interesting application of the results in this paper, we prove a density theorem of Arrow, Barankin, and Blackwell ( $[3,4])$.

First let us recall some notations:

Throughout the paper, $E$ is a separated locally convex topological linear space and $E^{*}$ its topological dual. $U(0)$ denotes the family of balanced open convex neighbourhoods of the origin in $E$. For $A \subset E$, $\operatorname{cone}(A), \operatorname{cl}(A)$, and $\operatorname{int}(A) \operatorname{denote}$ the generated cone, the closure, and the interior of $A$, respectively.

Let $C \subset E$ be a convex cone, and let $A$ be a nonempty subset of $E$. We say that $x \in A$ is an efficient point of $A$ with respect to $C$ if there exists $y \in A$, such that $y \in x-C$; then $y \in x+C$. Equivalently, $(x-C) \cap A \subset x+C$. If the $C$ is pointed (that is, $C \cap(-C)=\{0\}$ ), then $x \in A$ is an efficient point iff

$$
(x-C) \cap A=\{x\} .
$$

We denote by $E(A, C)$ the set of all efficient points of $A$ (with respect to $C$ ). We say that $x \in A$ is a proper efficient point of $A$ with respect to $C$ if there exists a closed convex cone $K \neq E$ such that $C \backslash\{0\} \subset \operatorname{int}(K)$ and $x \in E(A, K)$.

The set of proper efficient points of $A$ is denoted by $\operatorname{Prop} E(A, C)$. It is obvious that the set of proper efficient points of $A$ is contained in the set of efficient points,

$$
\operatorname{Prop} E(A, C) \subset E(A, C),
$$

but the converse is not generally true.

If $C$ is a convex cone, the convex set $B \subset C$ is said to be a base of $C$ if

$$
0 \notin \operatorname{cl}(B) \quad \text { and } \quad C=\operatorname{cone}(B)=\bigcup\{t B: t \geq 0\}=\{t b: t \geq 0, b \in B\} .
$$

A cone with base must be pointed.

Received by the editors December 14, 1993 and, in revised form, October 3, 1994. 1991 Mathematics Subject Classification. Primary 90C31.

Key words and phrases. Efficient point, proper efficient point, base of a cone, density. 
For the cone $C$ with base $B$, we define the "expansion" cone as below.

According to $0 \notin \mathrm{cl}(B)$, there exists a balanced convex open neighbourhood $U^{*} \in U(0)$ such that

$$
U^{*} \cap B=\emptyset .
$$

Let $N(0)=\left\{U \subset U^{*}: U \in U(0)\right\}$, and for $U \in N(0)$, let

$$
C_{U}=\operatorname{cl}(\operatorname{cone}(B+U)) \text {. }
$$

$C_{U}$ is said to be an expansion cone of $C$. It is clear that the expansion cone $C_{U}$ is a closed convex cone.

Lemma 1. For any $U \in N(0)$, we have that

$$
C \backslash\{0\} \subset \operatorname{int}\left(C_{U}\right) \text { and } 0 \notin \operatorname{int}\left(C_{U}\right) .
$$

Proof. Let $x \in C \backslash\{0\}$. There exists $b \in B, t>0$, such that $x=t b$, so

$$
x+t U=t b+t U \subset t(B+U) \subset \operatorname{cone}(B+U) \subset C_{U} .
$$

Therefore, $x \in \operatorname{int}\left(C_{U}\right)$.

Assume that $0 \in \operatorname{int}\left(C_{U}\right)$, for some $U \in N(0)$; then $C_{U}=E$. Choose any $b \in B$. By $-b \in C_{U}$, there is some net $\left\{\mu_{\tau}\left(b_{\tau}+u_{\tau}\right): \tau \in \Lambda\right\}, \mu_{\tau}>0, b_{\tau} \in B, u_{\tau} \in U$, such that $\mu_{\tau}\left(b_{\tau}+u_{\tau}\right) \rightarrow-b$, consequently $\mu_{\tau}\left(b_{\tau}+u_{\tau}\right)+b \rightarrow 0$. Since $U$ is a neighbourhood of the zero, there exists $\tau_{0} \in \Lambda$ such that

$$
\mu_{\tau_{0}}\left(b_{\tau_{0}}+u_{\tau_{0}}\right)+b \in U
$$

or

$$
\mu_{\tau_{0}}\left(b_{\tau_{0}}+u_{\tau_{0}}\right)+b=v_{\tau_{0}}, \quad v_{\tau_{0}} \in U
$$

and consequently

$$
\frac{\mu_{\tau_{0}}}{\mu_{\tau_{0}}+1} b_{\tau_{0}}+\frac{1}{\mu_{\tau_{0}}+1} b=\frac{1}{\mu_{\tau_{0}}+1} v_{\tau_{0}}+\frac{\mu_{\tau_{0}}}{\mu_{\tau_{0}}+1}\left(-u_{\tau_{0}}\right):=w_{\tau_{0}} .
$$

This implies $w_{\tau_{0}} \in B \cap U$ (since $U$ is balanced and convex, and $B$ is convex), which contradicts that $B \cap U=\varnothing$. This contradiction shows that $0 \notin \operatorname{int}\left(C_{U}\right)$, for all $U \in N(0)$. The proof of Lemma 1 is complete.

Theorem 1. Assume that $C$ is a closed convex cone with base B. Then for any compact set $A \subset E$, the set of proper efficient points of $A$ is dense in the set of efficient points of $A$,

$$
E(A, C) \subset \operatorname{cl}(\operatorname{Prop} E(A, C)) .
$$

Proof. Let $A \neq \emptyset$ be a compact set, and $x \in E(A, C)$. Without loss of generality, we may assume that $x=0$, thus $(0-C) \cap A=\{0\}$. We must show that there exists a net $\left\{x_{\tau}\right\} \subset \operatorname{Prop} E(A, C)$ such that $x_{\tau} \rightarrow 0$.

For any $U \in N(0)$, assume that $C_{U}$ is the expansion cone, and let $A_{U}=\left(0-C_{U}\right) \cap$ $A$. Since $A_{U}$ is compact, by using the usual existence theorem of efficient points (see [7, p. 140, Theorem 6.3]), there exists an efficient point of $A_{U}$ with respect to $C_{U}$. Let $x_{U} \in E\left(A_{U}, C_{U}\right)$. Since $A_{U}$ is a section of $A$ at $0, x_{U} \in E\left(A, C_{U}\right)$. Of course $C_{U}$ is a closed convex cone with $C \backslash\{0\} \subset \operatorname{int}\left(C_{U}\right)$, so

$$
x_{U} \in \operatorname{Prop} E(A, C) \text { for } U \in N(0) \text {. }
$$


Since $A$ is compact and $\left\{x_{U}: U \in N(0)\right\} \subset A$, without loss of generality, we may assume that $x_{U} \rightarrow x \in A$.

Now we have to prove that $x=0$.

Since $x_{U} \in A_{U}=\left(0-C_{U}\right) \cap A,-x_{U} \in C_{U}$. Hence there exists a net $t_{\tau}\left(b_{\tau}+u_{\tau}\right) \rightarrow$ $-x_{U}$, where $t_{\tau} \geq 0, b_{\tau} \in B, u_{\tau} \in U$. Considering $-x_{U}+U$ is a neighbourhood of $-x_{U}$, there exists some index $\tau_{U}$ such that

$$
t_{\tau_{U}}\left(b_{\tau_{U}}+u_{\tau_{U}}\right) \in-x_{U}+U
$$

or

$$
t_{\tau_{U}}\left(b_{\tau_{U}}+u_{\tau_{U}}\right)=-x_{U}+y_{U}, \quad y_{U} \in U .
$$

The number set $\left\{t_{\tau_{U}}: U \in N(0)\right\}$ must be bounded. Otherwise, we may assume that $t_{\tau_{U}} \rightarrow+\infty$. Obviously, the two nets $\left\{u_{\tau_{U}}: U \in N(0)\right\}$ and $\left\{y_{U}: U \in N(0)\right\}$ converge to 0 . From (1) follows that

$$
b_{\tau_{U}}=\frac{-x_{U}+y_{U}}{t_{\tau_{U}}}-u_{\tau_{U}} \rightarrow 0
$$

this implies $0 \in \operatorname{cl}(B)$, which is a contradiction, since $0 \notin \operatorname{cl}(B)$.

Since $\left\{t_{\tau_{U}}\right\}$ is a bounded number set, we may assume without loss of generality that $t_{\tau_{U}} \rightarrow t \geq 0$. By (1)

$$
t_{\tau_{U}} b_{\tau_{U}}=\left(-x_{U}+y_{U}\right)-t_{\tau_{U}} u_{\tau_{U}}=-x_{U}+\left(y_{U}-t_{\tau_{U}} u_{\tau_{U}}\right) \rightarrow-x .
$$

Considering $\left\{t_{\tau_{U}} b_{\tau_{U}}\right\} \subset C$, we conclude that $-x \in C$, or $x \in-C$. Since $0 \in$ $E(A, C)$, and $C$ is pointed, so $x \in(-C) \cap A=(0-C) \cap A=\{0\}$, therefore, $x=0$, consequently $x_{U} \rightarrow 0$. This completes the proof.

The result stated in Theorem 1 can be extended to weakly compact sets. Note that the symbol " $\rightarrow$ " denotes weak convergence.

Theorem 2. Let $C$ be a closed convex cone with bounded base B. Then for any weakly compact set $A \subset E$, the set of proper efficient points of $A$ is dense in the set of efficient points of $A$,

$$
E(A, C) \subset \operatorname{cl}(\operatorname{Prop} E(A, C)) .
$$

Proof. Let us replace strong convergence by weak convergence, and note that for a convex set $\mathrm{w}-\operatorname{cl}(\cdot)=\operatorname{cl}(\cdot)$. By using the same proof of Theorem 1, we can conclude that there exists a net $\left\{x_{U}: U \in N(0)\right\} \subset \operatorname{Prop} E(A, C)$ such that $x_{U} \rightarrow 0 \in E(A, C)$.

Now, we must prove that $x_{U} \rightarrow 0$. According to (1) in Theorem 1,

$$
t_{\tau_{U}}\left(b_{\tau_{U}}+u_{\tau_{U}}\right)=-x_{U}+y_{U} \quad \text { for } U \in N(0),
$$

where $u_{\tau_{U}} \rightarrow 0, y_{U} \rightarrow 0$, and $t_{\tau_{U}} \rightarrow t \geq 0$.

If $t>0$, then

$$
b_{\tau_{U}}=\frac{-x_{U}+y_{U}}{t_{\tau_{U}}}-u_{\tau_{U}} \rightarrow 0 .
$$

This implies $0 \in \mathrm{w}-\operatorname{cl}(B)=\operatorname{cl}(B)$, which is a contradiction. Therefore $t=0$, consequently $t_{\tau_{U}} \rightarrow 0$.

Since $B$ is bounded, $\left\{b_{\tau_{U}}\right\} \subset B$ is bounded. Therefore,

$$
x_{U}=y_{U}-t_{\tau_{U}}\left(b_{\tau_{U}}+u_{\tau_{U}}\right) \rightarrow 0 .
$$

The proof is complete. 
As an interesting application of the above theorems, we want to prove the ArrowBarakin-Blackwell theorem. For convenience, we introduce first some concepts and definitions.

Let $C$ be a closed convex cone, and let

$$
C^{\sharp}=\left\{f \in E^{*}: f(x)>0 \text { for all } x \in C \backslash\{0\}\right\} .
$$

An element $f \in E^{*}$ is said to be strictly positive if $f \in C^{\sharp}$. Let $A \neq \varnothing . \bar{x} \in A$ is said to be a positively proper efficient point of $A$ if there exists some strictly positive functional $f \in C^{\sharp}$ such that

$$
f(\bar{x}) \leq f(x) \text { for all } x \in A,
$$

i.e.

$$
f(\bar{x})=\min \{f(x): x \in A\} .
$$

We denote by $\operatorname{Ps} E(A, C)$ the set of those elements $\bar{x} \in A$ satisfying the above condition. Obviously, for any subset $A$, one has that

$$
\text { Ps } E(A, C) \subset E(A, C) \text {. }
$$

The converse is not true, but we can very simply show the following conclusions.

Theorem 3 (the Arrow-Barankin-Blackwell theorem [3-6]). (i) Let $C \subset E$ be a closed convex cone with base $B$. Then for any compact convex set $A \subset E$, the positively proper efficient point set of $A$ is dense in the efficient point set,

$$
E(A, C) \subset \operatorname{cl}(\operatorname{Ps} E(A, C)) .
$$

(ii) Let $C \subset E$ be a closed convex cone with bounded base $B$. Then for any weakly compact convex set $A$, the above result is true.

Proof. It is sufficient to show only that

$$
\operatorname{Prop} E(A, C) \subset \operatorname{Ps} E(A, C) \text {. }
$$

Indeed, let $\bar{x} \in \operatorname{Prop} E(A, C)$. By hypothesis, there exists a closed convex cone $K$ such that

$$
C \backslash\{0\} \subset \operatorname{int}(K) \quad \text { and } \quad \bar{x} \in E(A, K) .
$$

Since $\bar{x} \in E(A, K),(\bar{x}-K) \cap A \subset \bar{x}+K$. Consequently, we get $(\bar{x}-K \backslash l(K)) \cap A=\emptyset$, where $l(K)=K \cap(-K)$. Choose any $x \in \operatorname{int}(K)$. If $x \in l(K)$, then $x \in-K$, or $-x \in K$. This implies that $0=\frac{1}{2} x+\frac{1}{2}(-x) \in \operatorname{int}(K)$ (since $K$ is convex), which contradicts the assumption that $0 \notin \operatorname{int}(K)$. Therefore $x \in \operatorname{int}(K \backslash l(x))$. So, we obtain $\emptyset \neq \operatorname{int}(K) \subset \operatorname{int}(K \backslash l(K))$. Using a separating theorem, there exists $0 \neq f \in E^{*}$ and a real $t$ such that

$$
\begin{gathered}
\sup f(\bar{x}-K \backslash l(K)) \leq t \leq \inf f(A), \\
\sup f(\bar{x}-\operatorname{int}(K \backslash l(K))<t \leq \inf f(A) .
\end{gathered}
$$

As $\bar{x} \in A$ and $C \backslash\{0\} \subset \operatorname{int}(K) \subset \operatorname{int}(K \backslash l(K))$, the second inequality implies $f \in C^{\sharp}$. Furthermore, notice that for any $x \in K, \exists\left\{x_{n}\right\} \subset K \backslash l(K)$, such that $x_{n} \rightarrow x$, therefore, from the first inequality follows that sup $f(\bar{x}-K) \leq t \leq \inf f(A)$. By $0 \in K$, we have $f(\bar{x}) \leq f(x)$ for all $x \in A$, this is $\bar{x} \in \operatorname{Ps} E(A, C)$. So

$$
\text { Prop } E(A, C) \subset \operatorname{Ps} E(A, C) \text {; }
$$

this relation implies $\operatorname{cl}(\operatorname{Prop} E(A, C)) \subset \operatorname{cl}(\operatorname{Ps} E(A, C))$. 
Now, using Theorem 1 (respectively, Theorem 2), we obtain

$$
E(A, C) \subset \operatorname{cl}(\operatorname{Prop} E(A, C)) \subset \operatorname{cl}(\operatorname{Ps} E(A, C)) .
$$

The proof is complete.

\section{CONCLUding REMARKS}

Borwein in [4] proved that every efficient point of a weakly compact convex set in a finite-dimensional space is a limit of Borwein's properly efficient points. Using the concept of an approximating family of cones, and relaxing the convexity assumption imposed upon the objective set, Helbig [10] proved the above fact, too. In 1989, Sterna-Karwad discussed the existence of approximating families of cones in normed spaces, and using this concept, she showed that every efficient point of a weakly compact set in a normed space can be approximated by properly efficient points. Clearly, the approximating family of a cone cannot be extended onto topological vector spaces because this notion exists not in topological vector spaces (see [13]). In this paper, in order to derive the density theorem of properly efficient points in Luc's sense which is a generalization of Henig's properly efficient point in normed spaces, we consider a generalised concept of approximation families of cones, in which the "pointed" property is not required.

The author would like to thank the anonymous referee for many valuable suggestions and comments which improved the quality of this paper.

\section{REFERENCES}

1. D. T. Luc, Theory of vector optimization, Lecture Notes in Econom. and Math. Systems, vol. 319, Springer-Verlag, Berlin, 1989.

2. __ Recession cones and the domination property in vector optimization, Math. Programming 49 (1990).

3. K. J. Arrow, E. W. Barankin, and D. Blackwell, Admissible points of convex sets, Contributions to the Theory of Games, Vol. II (H. W. Kuhn and A. W. Tucker, eds.), Princeton Univ. Press, Princeton, NJ, 1953. MR 14:998h

4. J. M. Borwein, The geometry of Parete efficiency over cones, Math. Operations forsch. Statist. 11 (1980). MR 83f: 90110

5. J. Jahn, A generalization of a theorem of Arrow, Barankin, and Blackwell, SIAM J. Control Optim. 26 (1988). MR 89h:90224

6. W.-T. Fu, A note on the Arrow-Barankin-Blackwell theorem, J. Systems Sci. Math. Sci. (1994).

7. J. Jahn, Mathematical vector optimization in partially ordered linear spaces, Peter Lang, Frankfurt am Main, 1986. MR 87f:90095

8. G. R. Bitran and T. L. Magnanti, The structure of admissible points with respect to cone dominance, J. Optim. Theory Appl. 29 (1979), 573-614. MR 81e:90077

9. R. Hartley, On cone-efficiency, cone-convexity, and cone-compactness, SIAM J. Appl. Math. 34 (1978), 211-222. MR 58:7556

10. S. Helbig, Approximation of the efficient point set by perturbation of the ordering cone, $\mathrm{Z}$. Oper. Res. 35 (1991), 197-220. MR 92g:90138

11. M. Petschke, On a theorem of Arrow, Barankin, and Blackwell, SIAM J. Control Optim. 28 (1990), 395-401. MR 91e:90096

12. W. Salz, Eine topologische Eigenschaft der effizienten Punkte konvexer Mengen, Oper. Res. Verfahren 23 (1976), 197-202. MR 80b:90124

13. A. Sterna-Karwat, Approximation families of cones and proper efficiency in vector optimization, Optimization 20 (1989), 809-817. MR 91j:90067

Department of Mathematics, Nanchang University, Nanchang, Jiangxi, 330047, PeoPLE'S RePUBLIC OF CHINA 